\title{
Los delitos informáticos en la vulnerabilidad de las empresas en medios tecnológicos.
}

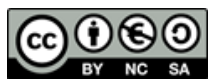

\section{Informatic crimes in the vulnerability of companies in technological media.}

Dalia Yasmin Ortiz Reinoso. ${ }^{1}$, Carlos Luis Pazmiño Palm. ${ }^{2}$, Luis Gabriel Pilay Salvatierr. ${ }^{3}$ \& Tito Hugo Ramos Soledispa. ${ }^{4}$

Recibido: 10-03-2019 / Revisado: 15-04-2019 /Aceptado: 30-05-2019/ Publicado: 14-06-2019

\begin{abstract}
.
DOI: https://doi.org/10.33262/cienciadigital.v3i2.6.598

The relationship with information and communication technologies (TIC`S), is of great importance at present for business development, however in Ecuador there is little investment in technological systems, due to the scarce economic resources and that it is necessary to invest is high for the implementation and the companies that implement it are by necessity of protection and speed of the information and many times without the securities, being vulnerable to computer frauds, for the accomplishment of the investigation the objective was to analyze the vulnerability to computer fraud in companies, conducting a field and exploratory research that facilitated a contact with the companies involved in the study, with a descriptive investigation, applying the survey instrument, and taking a representative sample, which provided the information relevant and supported the most appropriate analysis and decisions for the benefit of the companies and the employer, companies were chosen that comply with all legal and established parameters; in which a verification was carried out applying to a special digital forensic examination of the companies
\end{abstract}

\footnotetext{
${ }^{1}$ Universidad de Guayaquil. Guayaquil, Ecuador, dalia.ortizr@ug.edu.ec

${ }^{2}$ Universidad de Guayaquil. Guayaquil, Ecuador, carlos_pazmino2@hotmail.com

${ }^{3}$ Universidad de Guayaquil. Guayaquil, Ecuador, luis.pilays@ug.edu.ec

${ }^{4}$ Universidad de Guayaquil. Guayaquil, Ecuador, titoramos_ec@yahoo.com
} 
under study, which reflected the vulnerability to computer fraud that they have and that served as support to other companies depending on their sector.

Computer systems help Internal Control within the business sector, with the need to implement in organizations, processes and defined systems that allow evaluating the operations of a business, needs a clear support and consistent with the economic activity of each sector since these need different productive factors

keywords: Tic's, Companies, Computer Fraud, Vulnerability

\section{Resumen.}

La relación con las tecnologías de información y comunicación (TIC`S), es de gran importancia en la actualidad para el desarrollo empresarial, sin embargo en el Ecuador es poca la inversión en sistemas tecnológicos, debido a los recursos económicos que son escasos y lo que se debe invertir es alto para la implementación y las empresas que lo implementan es por necesidad de protección y rapidez de la información y muchas veces sin las seguridades, siendo vulnerables a fraudes informáticos, para el cumplimiento de la investigación se planteó como objetivo analizar la vulnerabilidad a fraudes informáticos de las empresas, realizando una investigación de campo y exploratoria que facilitó un contacto con la empresas involucradas objeto de estudio, con una investigación descriptiva, aplicando del instrumento de encuesta, y tomando una muestra representativa, la misma que proporcionó la información pertinente y sirvió de apoyo para el análisis y decisiones más adecuada en beneficio de las empresas y del empresario, se eligió empresas que cumpla con todos los parámetros legales y establecidos; en la cual se realizó una verificación aplicando a un examen especial y forense digital de la empresas objeto de estudio, donde se vio reflejado la vulnerabilidad a fraudes informáticos que poseen y que sirvió de apoyo a otras empresas dependiendo de su sector.

Los sistemas informáticos ayudan a un Control Interno dentro del sector empresarial, con la necesidad de implementar en las organizaciones, procesos y sistemas definidos que permitan evaluar las operaciones propias de un negocio, necesita de un soporte claro y coherente con la actividad económica de cada sector ya que estos necesitan de factores productivos diferentes.

Palabras Claves: Tic`S, Empresas, Fraude Informático, Vulnerabilidad 


\section{Introducción.}

Todas las instituciones del sector privado y público tienen la obligatoriedad de cuidar su información digital y con la ayuda de los avances tecnológicos según (LLC, 2017) a nivel mundial en Estados Unidos se evidencia en gran medida con la implementación de nuevas tecnología como videoconferencias holográfica que son muy realistas y ayuda en gran parte a los empresarios a mejorar las líneas de comunicación, todos esos avances traen consigo ventajas y desventajas, la mayor de ellas es que se encuentren vulnerabilidad de la información en los sistemas empresariales ocasionando los fraudes informáticos y perjuicios en contra del patrimonio y activos líquidos de las organizaciones.

Según (PWC, 2017) nos dice que América Latina es afectada en un $28 \%$ por delitos informáticos esto según informes proporcionados por la consultora Price Water house Coopers (PWC) a raíz de la última encuesta global que realizó sobre delitos económicos; el delito informático es progresivo conforme al desarrollo económico de los países. Adicionalmente según el último reporte proporcionado por el Observatorio de Delitos Informáticos de Latinoamérica (ODILA, 2017) del 100\% un 12,70\% corresponde a delitos de fraude o estafa informática destacando que es el segundo mayor delito de tipo informático que se realiza de manera más frecuente por los Hackers de Sombrero Negro.

Según (Ecured, 2012) de acuerdo a un estudio realizado se determinó que los costos directos relacionados con delitos informáticos que afectan a diversas personas en el mundo ascienden a \$ 110.000 billones de dólares en un periodo de doce meses; de igual manera el mismo estudio permite establecer que por cada segundo 18 adultos son víctimas de un delito informático. Según (INEC, 2016) nos refleja el porcentaje de personas que han utilizado internet en los últimos 12 meses por área, el 55,6\% de la población de Ecuador ha utilizado internet en los últimos 12 meses. En el área urbana el 63.8\% de la población ha utilizado internet, frente al 38.0\% del área rural. De acuerdo con estudios realizados por (INEC, 2016) indica que el equipamiento tecnológico del hogar a nivel nacional de cinco años se ha incrementado 13.7 puntos el equipamiento de computadoras portátiles en los hogares. Así también se refleja que el acceso al internet según área presenta el 36.0\% de los hogares a nivel nacional tienen acceso a internet, 13.5 puntos más que hace cinco años. 
Las empresas tienen conocimiento de la importancia de las tecnologías de información y comunicación dentro del desarrollo empresarial, y el aporte que genera en el rendimiento económico y financiero con su implementación en la parte operativa. Debe tener un adecuado sistema de control para sus ventas, ingresos económicos, cobros, control de efectivo empresarial, que estos no se controlen adecuadamente, pueda estar inmersos a fraudes informáticos y afectar a un manejo inadecuado de la tecnología necesaria para el cumplimiento de sus objetivos y la toma de decisiones. En los últimos años el Ecuador, los gobiernos han realizado inversiones en la adquisición de tecnología; para que las personas, instituciones públicas, privadas y empresas puedan acceder a este servicio, y no se ha limitado al empresario a la obtención de la misma contribuyendo a su disminuyendo su desarrollo, el inadecuado reclutamiento de personal por capacitación es deficiente sin la experiencia en esta área; esto genera un efecto negativo en la información de las bases de datos, es vulnerable desviando información e incluso provoca pérdidas económicas, limita el logro de los objetivos empresariales y el uso inadecuado a la información de la organización.

La investigación ha planteado como objetivo analizar la vulnerabilidad a fraudes informáticos de las empresas, acompañado objetivos específicos: Diagnosticar la problemática de la investigación en las empresas objeto de estudio aplicando una encuesta; Definir las bases teóricas bajo las cuales se sustenta la investigación en torno a vulnerabilidad de fraudes informáticos en las empresas; y analizar los niveles de vulnerabilidad en fraudes informáticos de las empresas.

La adquisición y aplicación de las TIC'S es de gran importancia en la actualidad para el sector empresarial, relacionado a la actividad que se realiza para la innovación de nuevos productos, desarrollo de estrategias, líneas de comunicación, optimización de procesos, análisis económico-financiero en donde se presentan vulnerabilidad a la información digital apareciendo los fraudes informáticos por la poca seguridad e importancia que dan los empresarios al tema.

\section{MARCO TEÓRICO REFERENCIAL}

De acuerdo a la institución gubernamental: Instituto Nacional Ecuatoriano de Censos INEC (2017), clasifica a las empresas por caracterices como: volumen de ventas anual y el número de personas que trabajan dentro de la empresas en las que se detalla en la Tabla 1 donde se 
observa cómo están clasificadas por el volumen es sus ventas anuales y las personas que la integran laboralmente;

Tabla 1 Clasificación de las empresas.

\begin{tabular}{lrrr}
\hline TIPO & DE & VOLUMEN DE & PERSONAS \\
EMPRESA & & VENTAS ANUALES
\end{tabular}

\begin{tabular}{lrc}
\hline Empresa grande & $\$ 5^{\prime} 000.001$ & 200 en adelante \\
\hline Mediana Empresa & $\$ 2^{\prime} 000.001$ a $\$$ & 100 a 199 \\
& $5^{\prime} 000.000$ & \\
\hline Pequeña Empresa & $\$ 100.001$ a $\$ 1^{\prime} 000.000$ & 10 a 49 \\
\hline microempresa & $<$ a $\$ 100.000$ & 1 a 9 \\
\hline
\end{tabular}

Fuente: (INEC, 2017).

Elaborado por: Grupo de trabajo.

Las empresas en Ecuador están clasificadas en relación con al sector económico donde se encuentran como lo son: el comercio de productos y servicios, la agricultura, el cultivo y explotación de bosques y montes, pesca, minería y construcción e industrias manufactureras (INEC, 2017). El conjunto de empresas define la actividad y el conjunto de actividades definen al sector, con una buena gestión empresarial se demuestra el crecimiento de sus ventas; cuando crecen las ventas; también crecen el sector, hay un desarrollo sostenible en la provincia y al mismo tiempo en el país (Padilla, Quispe, Rivera, Hernandez, \& Moreno, 2018).

Las empresas son unidades productivas que contribuyen económicamente a la sociedad, mismas que generan fuentes de trabajo siendo un factor importante para la economía del país, dentro de la clasificación de las PYMES se encuentran las grandes, medianas y pequeñas empresas que se diferencian por su patrimonio, personas que la constituyen y los productos o servicios que producen. Al analizar la vulnerabilidad de la información digital provoca como se indicó fraudes informáticos, frenando el desarrollo financiero de las empresas sin importar al sector que pertenezca, con la implementación de las TIC'S y muchas de las veces de última tecnología, sino podemos manejar el sistema, no podremos ser innovadores en los procesos internos y externos de la empresa, por más que apliquen estrategias empresariales, 
es decir, al tener tecnología acorde a las necesidades empresariales permite ser más eficiente y efectivo para alcanzar los objetivos planteados.

\section{Empresa}

Según (DEBITOOR, 2015) nos menciona el siguiente concepto de empresa:

"Una empresa es una unidad productiva agrupada y dedicada a desarrollar una actividad económica con ánimo de lucro. En nuestra sociedad, es muy común la creación continua de empresas. En general, una empresa también se puede definir como una unidad formada por un grupo de personas, bienes materiales y financieros, con el objetivo de producir algo o prestar un servicio que cubra una necesidad y por el que se obtengan beneficios. (párr.1)"

Por lo tanto, puedo decir que una empresa es una entidad conformada por personas que buscan enriquecer su capital mediante la realización de inversiones teniendo las capacidades técnicas y financieras para cumplirlo pretermitiendo así, dedicarse a la producción y transformación de productos y/o la prestación de servicios para satisfacer necesidades y deseos existentes en la sociedad.

\section{Teoría de la seguridad de la Información}

Proteger, salvaguardar y mitigar riesgos y amenazas es uno de los objetivos centrales de este tipo de teoría, es por ello que (Saruyón, 2014) menciona que la teoría de la información se basa en: "Todas aquellas medidas preventivas y reactivas del hombre, de las organizaciones y de los sistemas tecnológicos que permitan resguardar y proteger la información buscando mantener la confidencialidad, la disponibilidad e Integridad de la misma”.

La seguridad en la comunicación y difusión hoy por hoy se ha vuelto más vulnerable debido a los avances tecnológicos, es por ello por lo que hasta el momento la seguridad en la información sigue siendo un problema, que pocas empresas logran controlar. Proteger y cuidar la información en la actualidad es una tarea difícil. Teoría General de Ludwig Von Bertalanffy se considera fundamental la información y comunicación ya que parte de la idea de que una empresa es un sistema en el cual todos deben saber todo, he aquí la importancia del buen uso e implementación de herramientas de gestión de TIC que incidan en la mejora de los sistemas de evaluación y control interno, finalmente la teoría de seguridad informática 
hace hincapié en la difusión de información a nivel interno de la empresa y protección de la información a nivel interno y externo.

\section{Tecnología de Información y comunicación (TIC)}

Según (Ortí, 2015) acota: Que las TIC se desarrollan a partir de los avances científicos producidos en los ámbitos de la informática y las telecomunicaciones. Las TIC son el conjunto de tecnologías que permiten el acceso, producción, tratamiento y comunicación de información presentada en diferentes códigos (texto, imagen, sonido). El elemento más representativo de las nuevas tecnologías es sin duda el ordenador y más específicamente, Internet.

El sector en donde se desarrolla de forma amplia es la informática y los medios de telecomunicaciones, en el sentido de informática se puede mencionar que los elementos más utilizados son; el computador, tabletas y principalmente el internet que permite la conectividad y navegación, por otra parte, en términos de telecomunicaciones los elementos de mayor uso es el celular, líneas telefónica y medios de comunicación (televisión, radio, periódico), etc. La información presentada puede ser generada mediante imágenes, sonidos, letras, símbolos, etc. Todo este tipo de información es accesible para el usuario de forma inmediata al navegar en internet. Los medios de búsqueda de información mayormente utilizados y que han causado tendencia en los usuarios son: Facebook, WhatsApp, YouTube, Twitter y Skype.

Según (Ibanez \& Garcia, 2009) determina que las TIC también son: Herramientas computacionales e informáticas que procesan, almacenan, sintetizan, recuperan y presentan información representada de la más variada forma. Es un conjunto de herramientas, soportes y canales para el tratamiento y acceso a la información. Constituyen nuevos soportes y canales para dar forma, registrar, almacenar y difundir contenidos informacionales. (p.77). Emplear las TIC forma adecuada incrementa las posibilidades de conociendo y competitividad sea a nivel individual u organizacional. Las TIC facilitan la ejecución de tareas dentro y fuera de una organización o a también nivel personal, su uso varía de acuerdo con la actividad que realice la empresa, en la actualidad empresa que no invierte en TIC pierde competitividad en el mercado y su nivel de crecimiento va en forma decreciente. Las 
TIC es un arma de doble filo es por ello que las empresas deben establecer políticas se seguridad de información.

\section{Fraude informático}

Según (CORNELL, 2017) "El fraude cibernético e informático se refiere al fraude realizado a través del uso de una computadora o del Internet. La piratería informática (hacking) es una forma común de fraude: el delincuente usa herramientas tecnológicas sofisticadas para acceder a distancia a una computadora con información confidencial. Otra forma de fraude involucra la intercepción de una transmisión electrónica. Esto puede ocasionar el robo de la contraseña, el número de cuenta de una tarjeta de crédito u otra información confidencial sobre la identidad de una persona. (párr.1)”

Según (ALEGSA, 2010) nos dice que el fraude informático es "El delito informático implica cualquier actividad ilegal que encuadra en figuras tradicionales ya conocidas como robo, hurto, fraude, falsificación, perjuicio, estafa y sabotaje, pero siempre que involucre la informática de por medio para cometer la ilegalidad”. (párr.1)

\section{Vulnerabilidades de Seguridad Informática}

Según (CODEJOBS, 2012) "Una debilidad del sistema informático que puede ser utilizada para causar un daño. Las debilidades pueden aparecer en cualquiera de los elementos de una computadora, tanto en el hardware, el sistema operativo, cómo en el software". Podemos decir que la vulnerabilidad es la incapacidad de comunicación ocasionando efectos de un determinado cambio en su medio ambiente, ósea su inflexibilidad o incapacidad para adaptarse a ese cambio, que para la comunidad constituye un riesgo. Ser vulnerable a un fenómeno natural es ser susceptible de sufrir daño y tener dificultad de recuperase de ello.

\section{Metodología}

Para alcanzar el propósito central planteado la investigación se fundamenta en un paradigma critico propositivo, se enfoca y desarrolla bajo la modalidad cualitativo-cuantitativo, al ser cualitativo se refiere a los detalles y descripciones de los fenómenos que ocurren en el problema, la implementación de las TIC en las empresas y el grado seguridad que poseen los mismos para q no sean vulnerables, al ser cuantitativo se refiere a la objetividad como una manera de alcanzar el conocimiento, utilizando la medición exhaustiva y controlada, 
buscando la certeza del mismo, se utiliza una estrategia deductiva, basándose en la investigación bibliográfica y documental con procedimientos científicos, leyes, libros, revistas, e información oficial afines con los impuestos.

Al mencionar que es de carácter exploratorio ya que ayuda a examinar el tema de este estudio, aplicando un cuestionario tipo encuesta validado por especialistas en el tema sobre la vulnerabilidad de los delitos informáticos, el cual consta de 22 preguntas cerradas en escala de likert que estarán dirigidas a empresarios, administradores y contadores que tengan el conocimiento sobre la implementación de las TIC y su vulnerabilidad en los delitos informáticos donde se realizara el levantamiento de información, posteriormente se analizó los resultados obtenidos formando un conjunto de procedimientos que tienen como objetivo la producción de un meta-texto, de manera complementaria es de gran importancia entender sobre el tema planteado. La prueba estadística que se aplica es la correlación de Spearman, es una medida de la relación lineal entre dos variables aleatorias cuantitativas. A diferencia de la covarianza, la correlación de Pearson es independiente de la escala de medida de las variables. De manera menos formal, se define el coeficiente de correlación de Pearson como un índice que puede utilizarse para medir el grado de relación de dos variables siempre y cuando ambas sean cuantitativas.

\section{Resultados de las encuestas:}

\section{1. ¿A qué actividad comercial se dedica su empresa?}

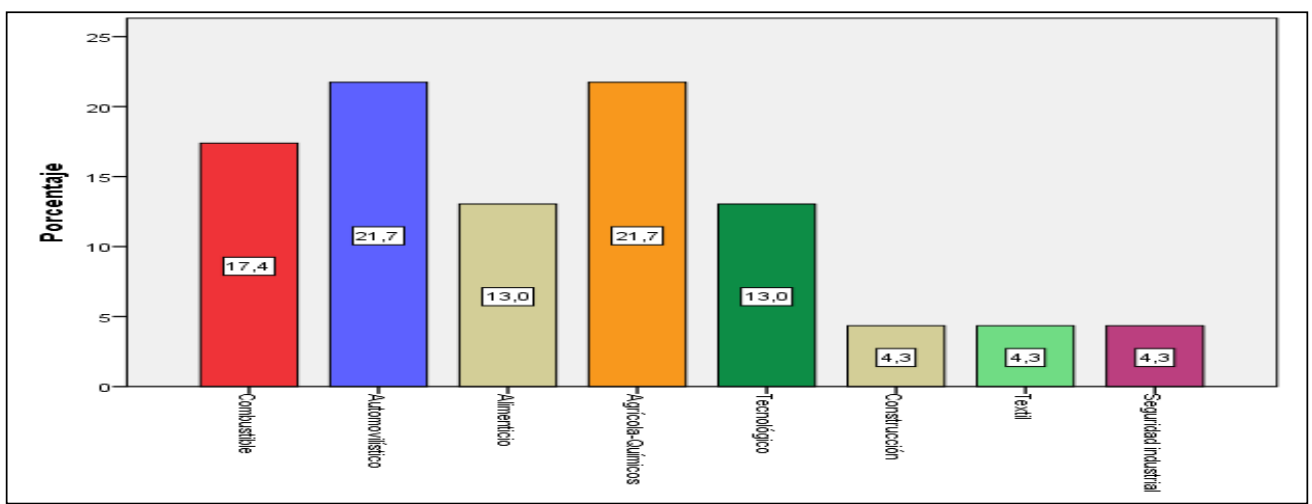

Imagen 1 ¿A qué actividad comercial se dedica su empresa?

Fuente: Encuesta

Realizado: Autora 


\section{Interpretación}

Las empresas de mayor influencia como se aprecia en la Imagen 1 ¿A qué actividad comercial se dedica su empresa?, se relacionada a la actividad agrícola en un $21.7 \%$ producción de alimentos y flores; ámbito automovilístico en un $21.7 \%$ con alta producción agrícola necesitan de maquinaria de riego; como para la movilización de mercadería; sector construcción, textil y seguridad industrial con menor representación con un $4.3 \%$ los mismos que aportan económicamente para el desarrollo de la actividad empresarial.

\section{2. ¿Cuántas computadoras posee su empresa?}

Del total de empresas encuestadas poseen en un rango de 0 a 5 equipos de cómputo reflejándose un porcentaje alto de 60.9\%; el 8.7\% tiene más de 20 computadores. Podemos decir que la mayor parte de las empresas pequeñas y medianas no poseen más de 5 computadoras para el desarrollo de sus actividades diarias; y las empresas grandes tienen mayor inversión en equipos tecnológicos al tener mayor número de trabajadores.

\section{3. ¿La Tecnología es un factor clave para la optimización de procesos?}

Tabla 1. ¿La Tecnología es un factor clave para la optimización de procesos?

\section{Dentro del ámbito empresarial al que pertenece su institución ¿La Tecnología es un factor clave para la optimización de procesos?}

Del

\begin{tabular}{|c|c|c|c|c|c|}
\hline & & Frecuencia & Porcentaje & $\begin{array}{c}\text { Porcentaje } \\
\text { válido }\end{array}$ & $\begin{array}{l}\text { Porcentaje } \\
\text { acumulado }\end{array}$ \\
\hline \multirow{3}{*}{$\begin{array}{l}\text { Válid } \\
\text { o }\end{array}$} & SI & 21 & 80,77 & 80,77 & 80,77 \\
\hline & $\mathrm{NO}$ & 5 & 19,23 & 19,23 & 100,0 \\
\hline & Total & 26 & 100,0 & 100,0 & \\
\hline
\end{tabular}

Fuente: Encuesta

Realizado: Autora

En la tabla 1 el total de encuestados el 80,77\% menciona que la tecnología si es un factor clave para la optimización de proceso, mientras que el 19,23\% menciona que la tecnología no es un factor clave para la optimización de procesos. Mayormente las empresas que consideran que la tecnología es parte primordial tanto para resguardar su información como 
para la optimización de procesos y recursos son las entidades de intermediación financiera, en menor proporción las empresas de salud, educación y turismo consideran que el factor clave no es tanto la tecnología sino el personal capacitado.

\section{4. ¿La utilización de paquetes informáticos como apoyo operativo en los procesos empresariales?}

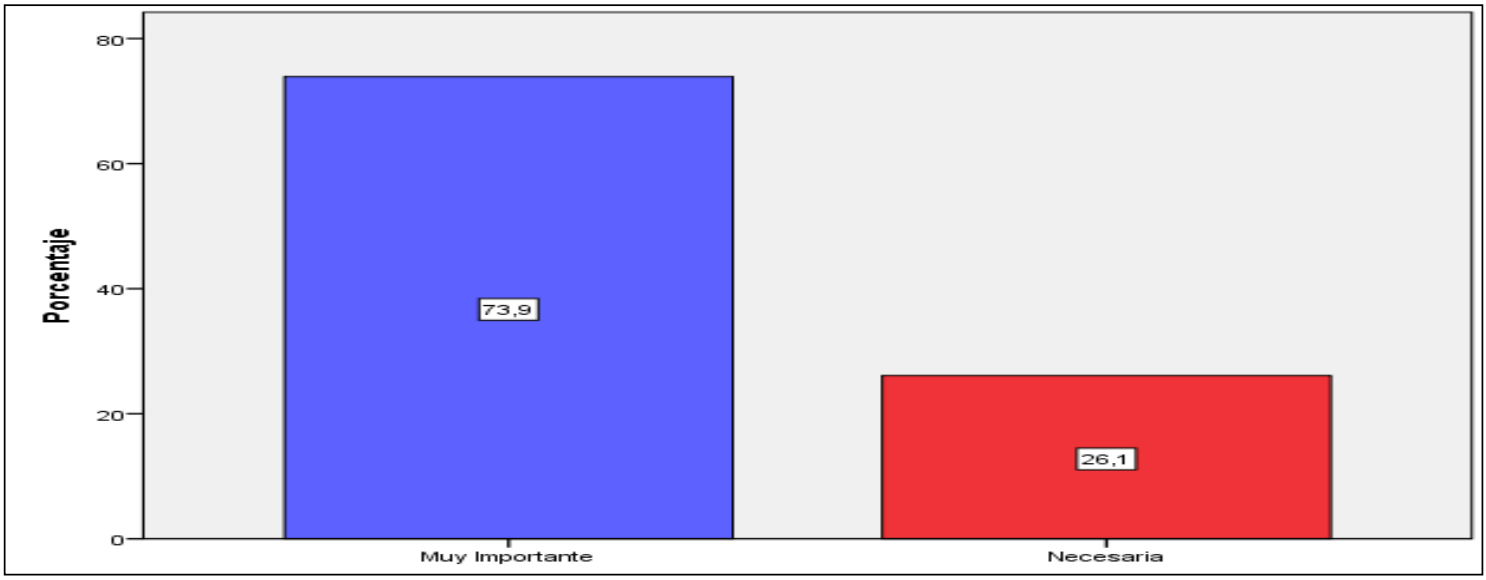

Imagen 2 ¿La utilización de paquetes informáticos como apoyo operativo? Fuente: Encuesta Realizado: Autora

Del total de las empresas encuestadas y podemos observar en la Imagen 2 ¿La utilización de paquetes informáticos como apoyo operativo?, un 73.9\% cree muy importante la utilización de paquetes informáticos para el desarrollo de los procesos y dependiendo del sector se encuentra enmarcado por la parte operativa y el manejo adecuado del mismo da rentabilidad empresarial, así como el logro de objetivos. Al tener seguridad de la información y mejor manejo se encuentran menos vulnerables para ataques cibernéticos. El $26.1 \%$ dice que es necesaria el buen uso de los paquetes informáticos teniendo en cuenta la necesidad de este, pero limitándose a los recursos que posee cada empresa.

\section{DISCUSIÓN}

Del análisis de los resultados se pudo comprobar que los fraudes informáticos ante el contexto, no incide en los resultados económicos de las empresas, conocen de la importancia de las tecnologías de la información, sin embargo, tienen limitaciones en cuanto a los recursos económicos que se requiere para la implementación. En nuestro estudio las grandes 
empresas cuentan con un adecuado sistema tecnológico que salvaguarda la información, teniendo resultados eficientes para el funcionamiento de su entidad.

En cuanto a las medianas y pequeñas empresas, no se ven en la necesidad de implementar avanzadas tecnologías destinadas a la protección de datos, de acuerdo con los requerimientos como al volumen de su negocio. Las restricciones en los sistemas operativos ya sea grande o pequeña empresa es de importancia, en el proyecto se evidencia que el fraude informático que genera mayor debilidad empresarial son los virus mediante enlaces o correos maliciosos y el inapropiado uso de los equipos tecnológicos por parte de los empleados.

Con relación a inversión en TIC y el número de empleados si importa el tamaño de empresa como la cantidad de empleados con la cuenta incide en el tipo de inversión que realice en tecnologías de la información y comunicación. En el sector comercial está limitada la inversión en TIC por los costos elevados sin embargo en los últimos 5 años, los resultados obtenidos para las pequeñas y medianas empresas radica una inversión en el rango de 1000 a 5000 dólares en un porcentaje de $17.4 \%$, el $21.7 \%$, las grandes empresas realizan inversiones altas en valores que asciende a más de 20000 dólares con un porcentaje minoritario del $4.3 \%$. Esto se debe al volumen de ventas, además que requieren ser más competitivos en el mercado.

\section{Bibliografía}

1. ALEGSA. (05 de 12 de 2010). Diccionario de informática. Obtenido de http://www.alegsa.com.ar/Dic/delito_informatico.php

2. CODEJOBS. (07 de 09 de 2012). Seguridad Informática: ¿Qué es una vulnerabilidad, una amenaza y un riesgo? Obtenido de https://www.codejobs.biz/es/blog/2012/09/07/seguridad-informatica-que-es-unavulnerabilidad-una-amenaza-y-un-riesgo

3. CORNELL. (18 de 08 de 2017). Fraude Cibernético e Informático. Obtenido de https://www.law.cornell.edu/wex/es/fraude_cibern\%C3\%A9tico_e_inform\%C3\%A1tic o

4. DEBITOOR. (14 de 05 de 2015). Què es una empresa. Obtenido de https://debitoor.es/glosario/definicion-empresa

5. Ecured. (2012). ECURED. Recuperado el 2017 de 03 de 01, de ECURED: https://www.ecured.cu/Ataque_inform\%C3\%A1tico 
6. ECURED. (19 de 09 de 2017). Tecnologías de la Información y las comunicaciones. Obtenido de https://www.ecured.cu/Tecnolog\%C3\%ADas_de_la_informaci\%C3\%B3n_y_las_com unicaciones

7. Ibanez, P., \& Garcia, G. (2009). Utiliza las tecnologias de infromación y Comunicación. México: Cengage Learning Editores S.A. Recuperado el 22 de Noviembre de 2017

8. INEC. (2 de 12 de 2016). Tecnologías de la Informacion y Comunicación TIC'S. Obtenido de http://www.ecuadorencifras.gob.ec/documentos/webinec/Estadisticas_Sociales/TIC/2016/170125.Presentacion_Tics_2016.pdf

9. INEC. (1 de Febrero de 2017). INEC. Obtenido de INEC.EC: http://www.ecuadorencifras.gob.ec/documentos/webinec/Estadisticas_Economicas/DirectorioEmpresas/Empresas_2014/Principales_Result ados_DIEE_2014.pdf

10. LLC. (02 de 10 de 2017). Avances Tecnológicos a Nivel Mundial. Obtenido de https://avancestecnologicosanivelmundial.wikispaces.com/Nuevos+Avances+Tecnol\% C3\%B3gicos+en+EE.UU

11. ODILA. (14 de 08 de 2017). Observatorio de Delitos Informáticos de Latinoamérica. Obtenido de https://www.odila.org/

12. Ortí, C. B. (28 de Septiembre de 2015). www.uv.es. Recuperado el 22 de Noviembre de 2017, de www.uv.es: http://www.uv.es/ bellochc/pdf/pwtic1.pdf

13. Padilla, M., Quispe, A., Rivera, N. D., Hernandez, A., \& Moreno, G. K. (2018). El emprendimientocomo gestión empresarial para un desarrollo sostenible. scielo, 1.

14. PWC. (14 de Agosto de 2017). Delitos Informáticos. Obtenido de http://www.pwc.es/

15. Saruyón, R. (24 de Abril de 2014). Informàtica y tecnologìa. Recuperado el 28 de Diciembre de 2017, de http://informaticacae.blogspot.com: http://informaticacae.blogspot.com/2014/04/8-teoria-seguridad-informatica.html

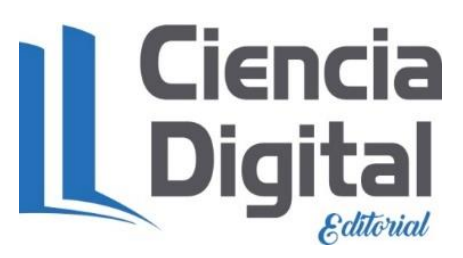




\section{PARA CITAR EL ARTÍCULO INDEXADO.}

Pazmiño Palm, C., Ortiz Reinoso, D., Pilay Salvatierr, L., \& Ramos Soledispa, T. (2019). Los delitos informáticos en la vulnerabilidad de las empresas en medios tecnológicos. Ciencia Digital, 3(2.6), 481-494. https://doi.org/10.33262/cienciadigital.v3i2.6.598

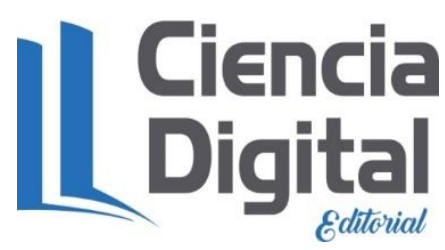

El artículo que se publica es de exclusiva responsabilidad de los autores y no necesariamente reflejan el pensamiento de la Revista Ciencia Digital.

El artículo queda en propiedad de la revista y, por tanto, su publicación parcial y/o total en otro medio tiene que ser autorizado por el director de la Revista Ciencia Digital.
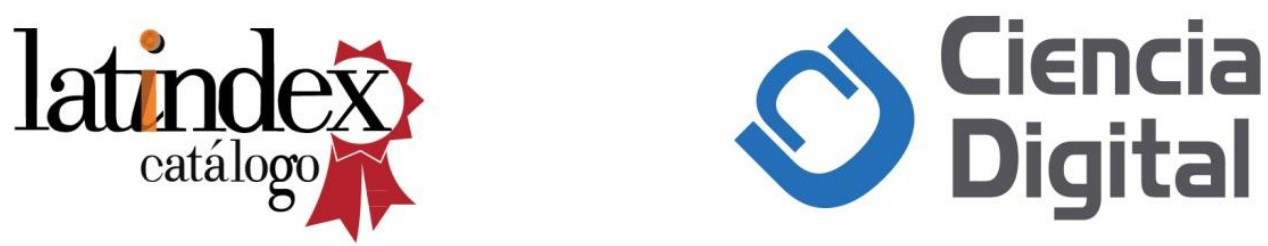